\title{
OPEN A strain of Phoma species improves drought tolerance of Pinus tabulaeformis
}

\author{
Xiu Ren Zhou ${ }^{1 凶}$, Lei Dai ${ }^{1,2}$, Gui Fang Xu' ${ }^{1}$ \& Hong Sheng Wang ${ }^{1}$
}

Global warming has led to frequent droughts, posing challenges for afforestation in arid and semiarid regions. In search of labor-saving and money-saving methods to improve the survival and growth rates of trees in these regions, we isolated and identified fungal endophytes that can potentially enhance the drought-resistance abilities of seedlings. We isolated 93 endophytic strains from the roots of $P$ inus tabulaeformis trees grown on an arid cliff. Three isolates increased the drought resistance of the tree seedlings. Using morphological, molecular, and physiological-biochemical methods, we identified three isolates as different clones of a strain of Phoma spp. and studied the strain's effect on stress resistance-related substances in the seedlings. The results showed that the strain improved drought tolerance and increased the seedlings' proline levels and antioxidant enzyme activities. The strain also secreted abundant extracellular abscisic acid, which likely triggered the seedlings' protective mechanisms. This endophytic strain may provide a cheaper labor-saving, sustainable alternative to traditional methods of enhancing drought resistance.

Climate change and global warming cause frequent drought, presenting a serious threat to tree survival and growth ${ }^{1,2}$, which severely limits the development of artificial afforestation and environmental management in arid areas. Some sustainable, labor-saving, and money-saving alternatives to traditional methods, such as artificial irrigation and water retention agents, have become necessary. Much research has suggested that endophytic fungi can improve the ability of woody plants to resist drought stress. Therefore, screening for potential endophytes enhancing plant drought resistance may be a crucial step in finding a promising alternative to traditional methods.

Plants usually respond to drought stress through a series of morphological, physiological, and biochemical strategies, such as increasing their root biomass, decreasing their chlorophyll content, photosynthesis, stomatal conductance and leaf area, closing their stomata, and enhancing antioxidant activities ${ }^{3-6}$. Plant hormone levels also change, especially that of abscisic acid (ABA), which initiates protective mechanisms to protect plants from drought damage ${ }^{7,8}$. Endophytes can alter the morphological, physiological, and biochemical characteristics of plants to enhance their ability to adapt to a drought ecological environment ${ }^{9-11}$.

Endophytes can improve the host plant resistance to drought stress based on different mechanisms ${ }^{9,12,13}$. Some endophytes can increase the biomass, length, and number of host roots to enhance the ability of a plant to absorb water, thus improving its drought resistance ${ }^{14-17}$. Previous studies showed that inoculating gramineous plants with corresponding endophytic fungi significantly increased the plant tiller numbers, aboveground biomass, and leaf number, showing enhanced drought resistance compared with noninoculated plants ${ }^{18,19}$. Murphy et al. found that under drought stress, an endophytic fungus taken from Hordeum murinum subsp. murinum enabled the barley to preferentially allocate resources to its aboveground parts to achieve more aboveground biomass compared with uninoculated plants ${ }^{20}$. Under drought conditions, hybrid poplars inoculated with endophytic communities increased their antioxidant activities to achieve higher drought resistance than uninoculated plants ${ }^{21}$. Endophytic fungi improved the gas exchange, chlorophyll content, photosynthesis, and chloroplast fluorescence in both red oak and Sorghum bicolor seedlings to respond to drought stress ${ }^{16,17,22,23}$. Increased metabolism of amino acids, proteins, and other secondary substances has also been related to drought-resistance responses in some inoculated plants ${ }^{22,24}$. Levels of hormones, such as ABA, in some inoculated plants can be adjusted to improve their drought resistance ${ }^{25-27}$. Endophytes can also adjust a plant's osmotic ability via active accumulation of organic and inorganic solutes (e.g., soluble sugars, glycine betaine, organic acids and proline) in the host tissue, thereby enhancing the plant's drought resistance ${ }^{28-31}$. Therefore, an understanding of the mechanisms 


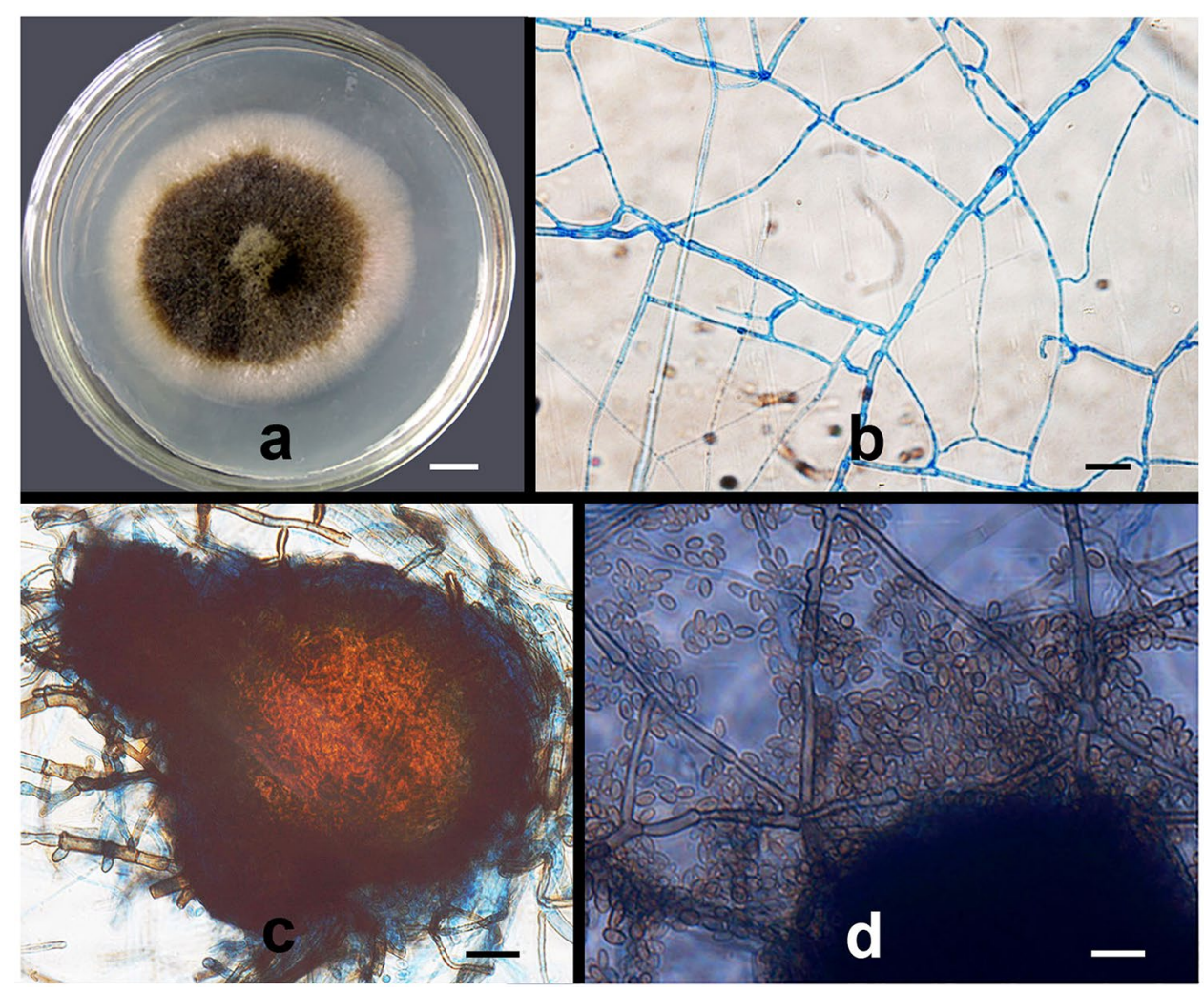

Figure 1. Morphological characteristics of strain PTD37 cultivated on PDA medium. (a) A colony after cultivation at $22-23^{\circ} \mathrm{C}$ for 10 days. (b) Septate hyphae and branches. (c) Pycnidium. (d) Conidia. Scale bars: (a) $=1.0 \mathrm{~cm} ;(\mathbf{b}-\mathbf{d})=10 \mu \mathrm{m}$.

by which screened potential endophytes enhance plant drought resistance will promote the development and utilization of these endophytes.

In this study, we hypothesized that when Pinus tabulaeformis, a tree widely cultivated in northern and northwestern China, grows under extreme drought conditions, its roots are parasitized by endophytic fungi that can improve its drought resistance. Potential endophytic fungi strengthening P. tabulaeformis drought resistance were screened. The effects of these endophytes on seedling antioxidant activity, ABA content, and other physiological characteristics were measured and estimated. Finally, potential endophytic fungi improving plant drought resistance were obtained, and the mechanism through which they enhanced plant drought resistance was speculated.

\section{Results}

We obtained 93 endophytic fungal isolates from Chinese pine roots. Screening of the drought-resistant endophytes revealed that all pine needles of the seedlings treated with fermentation broth supernatant (FBS) from strains PTD37, PTD56, and PTD78 remained green and tough after the application of 25\% polyethylene glycol 6000 (PEG6000) solution. However, the other seedlings' early needles gradually lost their green color and became softer after PEG6000 application, suggesting that the seedlings watered with FBS from PTD37, PTD56, and PTD78 exhibited significantly improved drought-resistance abilities. The colonies, hyphae, ascospores, and perithecia of the three isolates all had the same morphological characteristics. The isolates also had identical nucleotide sequences in their internal transcribed spacer (ITS) regions (accession numbers: MN686446, MN686446, and MN686448) and their 18S rDNA (accession numbers: MN698959, MN698960, and MN698961). Therefore, the three isolates were likely clones from the same strain, and PTD37 was further studied and analyzed as a representative of the three isolates.

Morphological and molecular analyses indicated that PTD37 was a species of Phoma. The PTD37 strain colonies grew slowly on potato dextrose agar (PDA) medium at $27-28^{\circ} \mathrm{C}$. The colonies were initially slightly almond colored; however, after 6 days, the colony centers became brown, while the margins remained slightly almond. The colony diameter averaged $5-6 \mathrm{~cm}$ on day 10 (Fig. 1a). The hyphae $(0.5-3.0 \mu \mathrm{m}$ wide) had typical septae, with a $>45^{\circ}$ branching angle (Fig. 1b). Brownish black pycnidia $(80-120 \times 70-100)$ were produced during mycelial development (Fig. 1c). When the pycnidia matured, brown spores $(4.5-6 \times 2.5-3.0 \mu \mathrm{m})$ were released from the rupture once the perithecia were crushed and broken (Fig. 1d). These morphological features were similar to those of Phoma spp. described in the literature ${ }^{32,33}$. A comparison of the ITS sequence of PTD37 with entries in GenBank showed that the maximum score, total score, E-value, query cover, and percentage identity between the first three hits (accession numbers: MN944409.1, FJ755260.1, and FJ755259.1) and PTD37's ITS were $907-909,907-909,99 \%, 0.0$, and $99.40-99.60 \%$, respectively. The sequences from the first three hits were from 


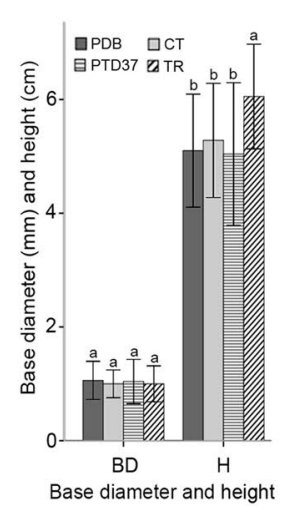

a

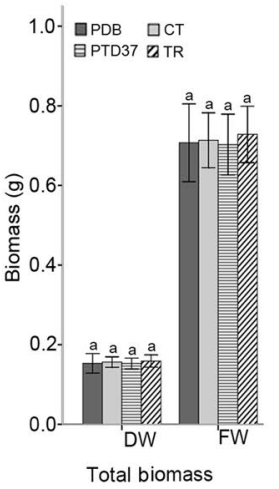

b

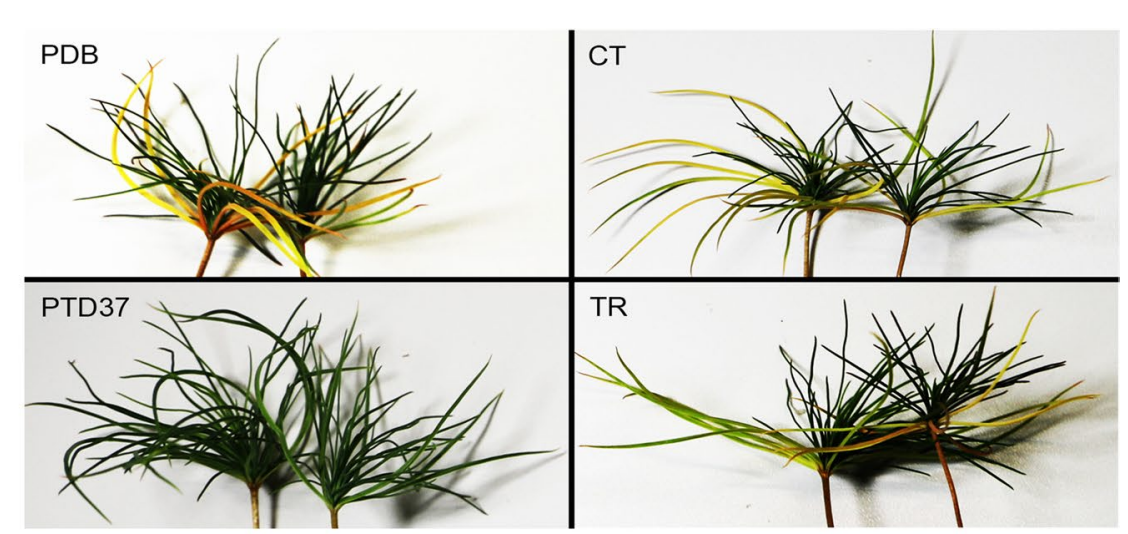

C

Figure 2. Effect of different treatments on total biomass and seedling phenotype before PEG application. (a) Effect of different treatments on the basal diameter and height of seedlings before PEG application. (b) Effect of different treatments on the total biomass of seedlings before PEG application. (c) Vegetative appearances of different treatment seedlings 15 days after PEG application, showing the seedlings treated by PTD37 with green and tough leaves but other seedlings with a loss of green color and wilted lower leaves. $B D$ basal diameter, $H$ height, $C T$ control, $D W$ dry biomass, $F W$ fresh weight, $P D B$ potato dextrose broth, PTD37 fermentation broth supernatant of the PTD37 strain, TR fermentation broth supernatant of Trichoderma harzianum. In (a) and (b), data are the means \pm SD from five independent tests. Different letters on error bars indicate significant differences at $P=0.05$ between the analyzed traits of seedlings from different treatments before PEG application. Scale bars: $(\mathbf{c})=5.0 \mathrm{~cm}$.

Didymella macrostoma, Phoma medicaginis strain CZ323, and Phoma medicaginis strain CZ316B. The PTD37 18S sequence comparison against known sequences in GenBank showed that the first three hits (accession numbers: KX519725.1, MT649541.1, and MT150595.1) were Didymella macrostoma isolate MS-2, Didymella macrostoma isolate 48, and Phoma sp. strain. The maximum scores, total scores, E-value, query cover, and percentage identity between the $18 \mathrm{~S}$ of the first three hits and that of PTD37 were 913-918, 913-918, 99\%, 0.0, and 99.02-99.41\%, respectively. Therefore, the PTD37 isolate was identified as Phoma spp.

After application of the PTD37 FBS to the seedlings, their total biomass, height, and ground diameter did not significantly differ from those of the other treatments except the Trichoderma harzianum FBS treatment (Fig. 2a,b). After treatment with T. harzianum FBS, the seedlings were significantly taller than the other seedlings. However, all seedlings had similar total biomasses and ground diameters (Fig. 2a,b). After application of PEG6000 solution for 15 days, the seedlings pretreated with PTD37 FBS had green and tough leaves, whereas the lower leaves of the seedlings that received the other treatments were yellow-green, yellow, or brown and softer (Fig. 2c).

PTD37-treated seedlings had a significantly higher proline level than the seedlings in the potato dextrose broth (PDB) and distilled water (CT) treatments before PEG application, but there was no significant difference between PTD37 and TR. Application of PTD37 FBS significantly increased the seedlings' proline levels compared with those of the other seedlings after PEG application (Fig. 3a). The peroxidase (POD), catalase (CAT), and superoxide dismutase (SOD) activities of the PTD37-treated plants were more robust than those of the plants that received the other treatments during the measurement period (Fig. 3b-d). Once treated with PEG solution, the proline content and antioxidant enzyme activities of all seedlings increased rapidly over the first 6 days, especially over the first 3 days (Fig. 3). Treatment with PEG gradually increased the difference in the proline content and antioxidant enzyme activity between the PTD37-treated seedlings and the other seedlings (Fig. 3). These levels also reached or neared the maximum on day 6 after PEG application but only increased slightly or did not change from days 6-9.

Before PEG application, no seedlings differed in their chlorophyll or leaf moisture content, but they did differ in their malonic dialdehyde (MDA) content (Fig. 4). However, after PEG application, the PTD37-FBS-treated seedling leaves had higher chlorophyll and water contents and lower MDA levels than the leaves of the other treatments. Thus, PTD37-FBS-treated seedlings could better withstand drought-stress conditions (Fig. 4). After PEG application, the chlorophyll content in the leaves of the seedlings treated with T. harzianum FBS was higher than that in those treated with distilled water or PDB FBS but was significantly lower than that in the PTD37-FBStreated seedling leaves (Fig. 4b). The MDA, chlorophyll, and water contents in all treatments stopped changing 6-9 days after PEG application (Fig. 4a).

The PTD37-FBS-treated seedlings had higher ABA levels than the other seedlings before PEG application. However, the ABA content of the PTD37-treated seedlings increased significantly slower than that of the control, PDB-treated or CT-treated seedlings on day 1 after PEG treatment (Fig. 5a), indicating lower stress perception in the PTD37-treated plants owing to their better stress resistance. ABA levels in the PTD37-treated plants became progressively similar to those of the other plants from day 3 of PEG application (Fig. 5a). The ABA level in the PTD37 FBS was approximately 7 times that of the T. harzianum FBS. Gibberellin (GA) 3 was also significantly higher in the PTD37 FBS than in the T. harzianum FBS. The auxin level of T. harzianum FBS was 7 times that of PTD37 FBS. T. harzianum FBS had a significantly higher level of GA7 than PTD37 FBS. However, these two FBSs had similar GA1 contents (Fig. 5b). 


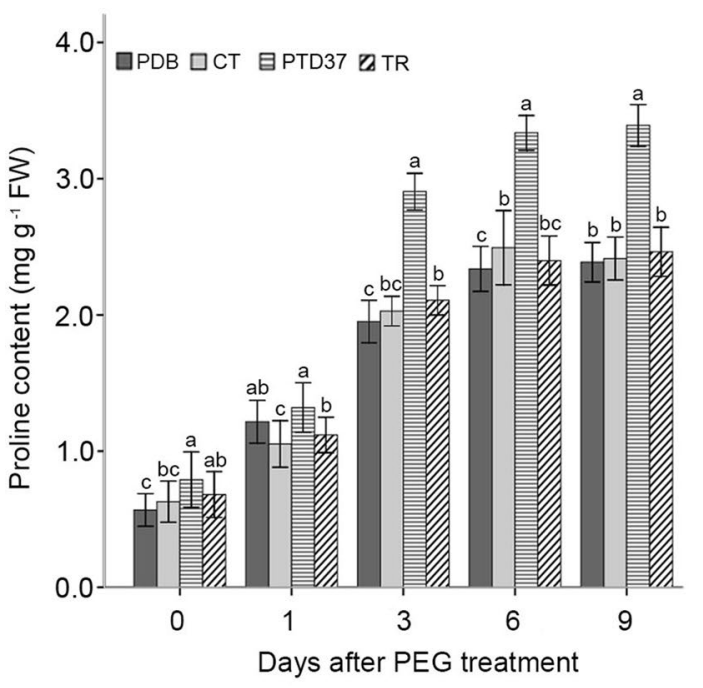

a

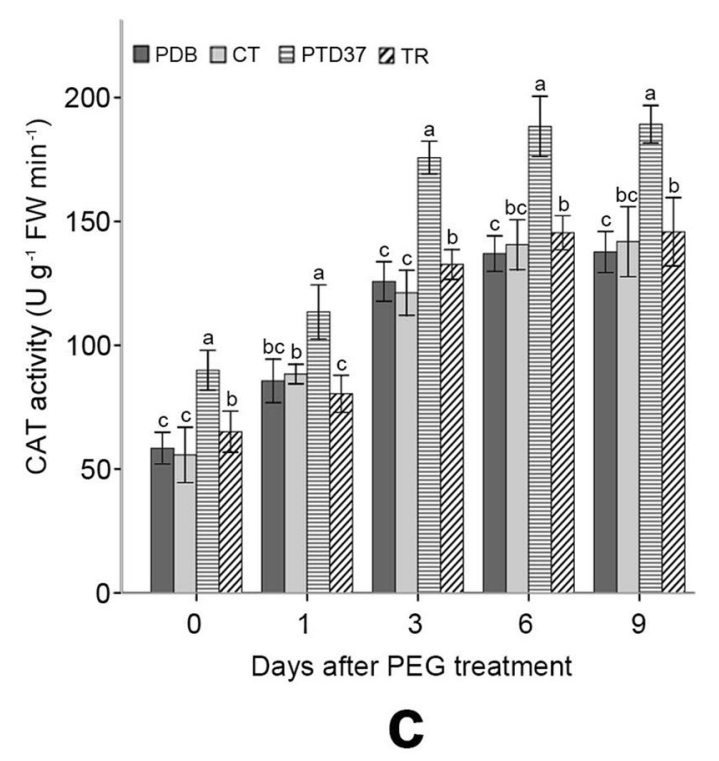

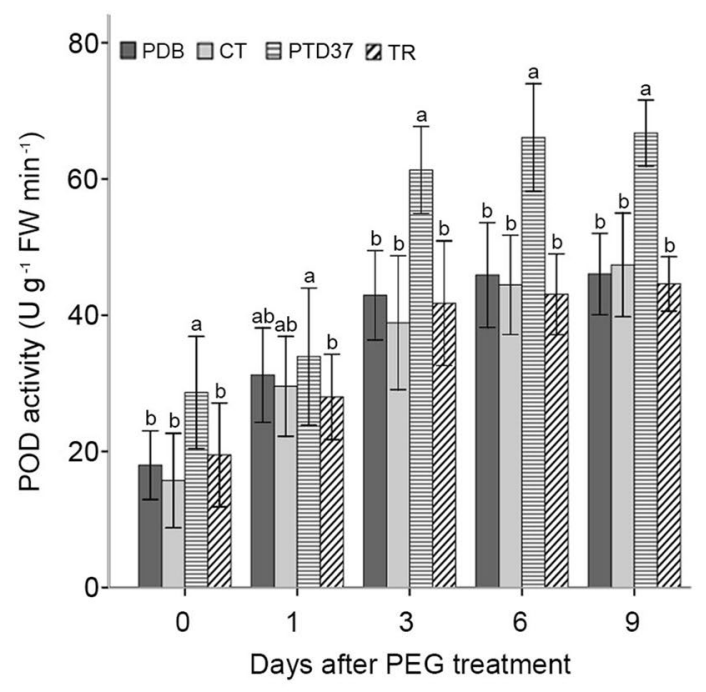

b

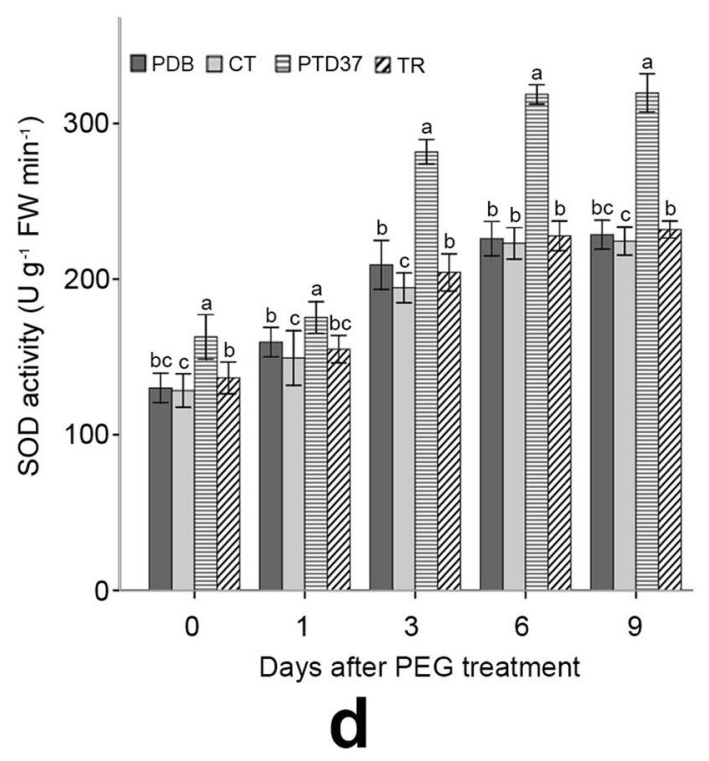

Figure 3. Effect of different treatments on the proline content and POD, CAT and SOD activities of seedling leaves before and after PEG application. (a) Effect of PEG application on the leaf proline content of seedlings under different treatments. (b) Effect of PEG application on the leaf POD activity of seedlings under different treatments. (c) Effect of PEG application on the leaf CAT activity of seedlings under different treatments. (d) Effect of PEG application on the leaf SOD activity of seedlings under different treatments. CT = control; $\mathrm{PDB}=$ potato dextrose broth; PTD37 = fermentation broth supernatant of the PTD37 strain; TR = fermentation broth supernatant of $T$. harzianum. Data are the means \pm SD from five independent tests. Different letters on error bars indicate significant differences at $P=0.05$ between analyzed traits of seedlings from different treatments on the same day after PEG application. The values on day 0 after PEG treatment indicate that they were measured before PEG application.

\section{Discussion}

To ensure the survival of $P$. tabulaeformis in semiarid and arid areas, irrigation and water retention agents require substantial financial resources, which severely limits artificial afforestation and environmental management. Endophytic fungi that improved host plant drought resistance are considered to be a sustainable, labor-saving, and money-saving approach. Whether $P$. tabulaeformis is parasitized by fungal endophytes, enhancing its drought resistance, remains unclear. The screening of potential endophytes enhancing the ability of $P$. tabulaeformis to resist drought and research on the interaction mechanism between them will facilitate the development of an alternative to traditional methods.

In this study, we isolated a fungal endophyte from the roots of $P$. tabulaeformis, which can potentially improve the ability of this species to resist drought. Previous researchers screened a variety of fungal endophytes that can improve plant drought resistance. These endophytes may belong to different fungal taxa. Based on morphological 


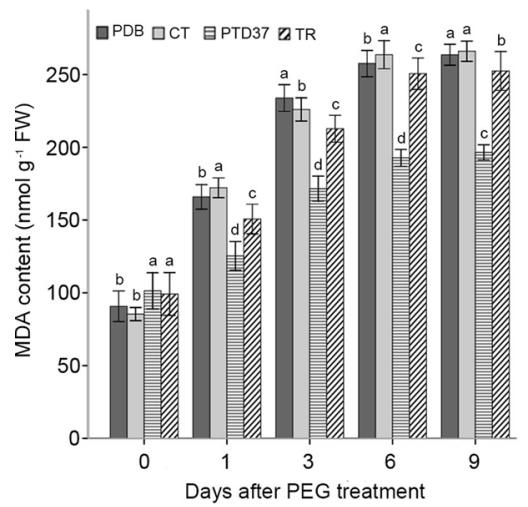

a

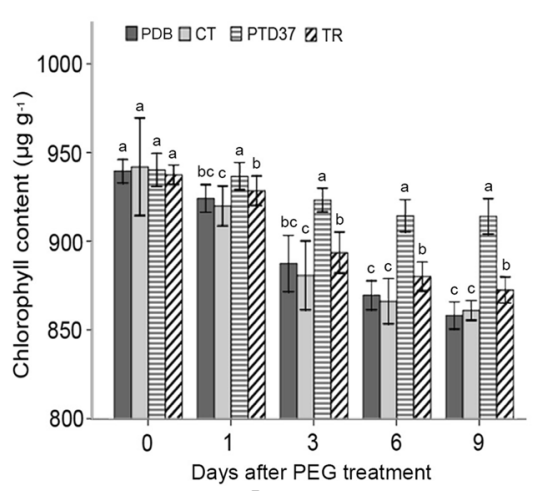

b

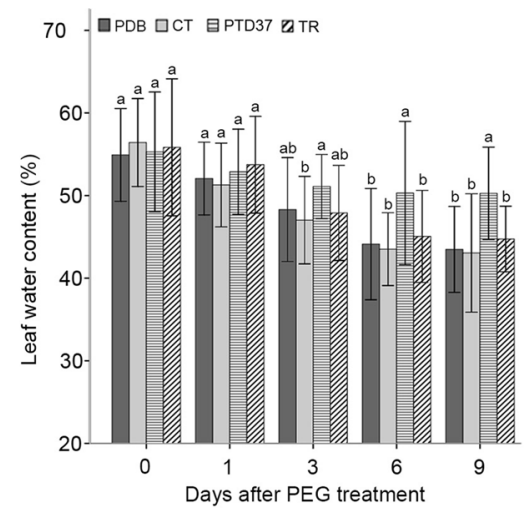

C

Figure 4. Effect of different treatments on the MDA, chlorophyll, and water contents of seedling leaves before and after PEG application. (a) Effect of PEG application on the leaf MDA content of seedlings under different treatments. (b) Effect of PEG application on the leaf chlorophyll content of seedlings under different treatments. (c) Effect of PEG application on the leaf water content of seedlings under different treatments. PDB potato dextrose broth, PTD37 fermentation broth supernatant of the PTD37 strain, TR fermentation broth supernatant of $T$. harzianum. Data are the means \pm SD from five independent tests. Different letters on error bars indicate significant differences at $P=0.05$ between the analyzed composition contents of seedlings from different treatments on the same day after PEG application. The values on day 0 after PEG treatment indicate that these values were measured before PEG application.

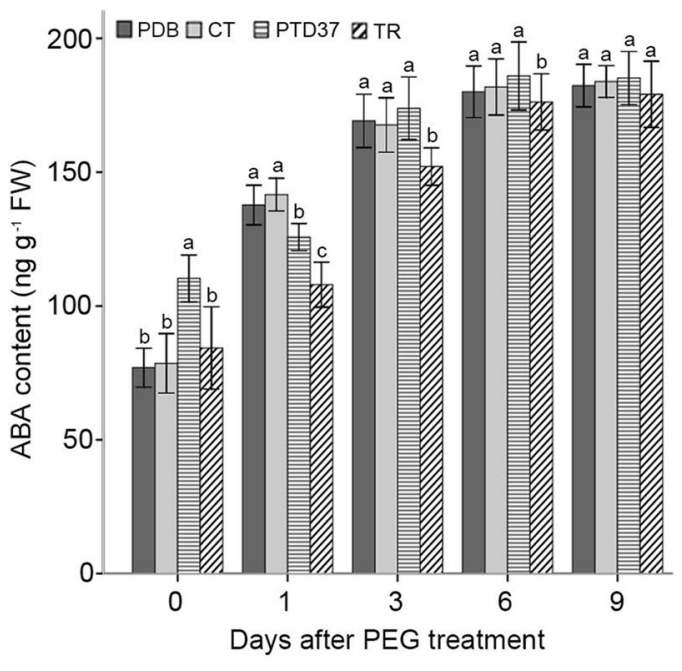

a

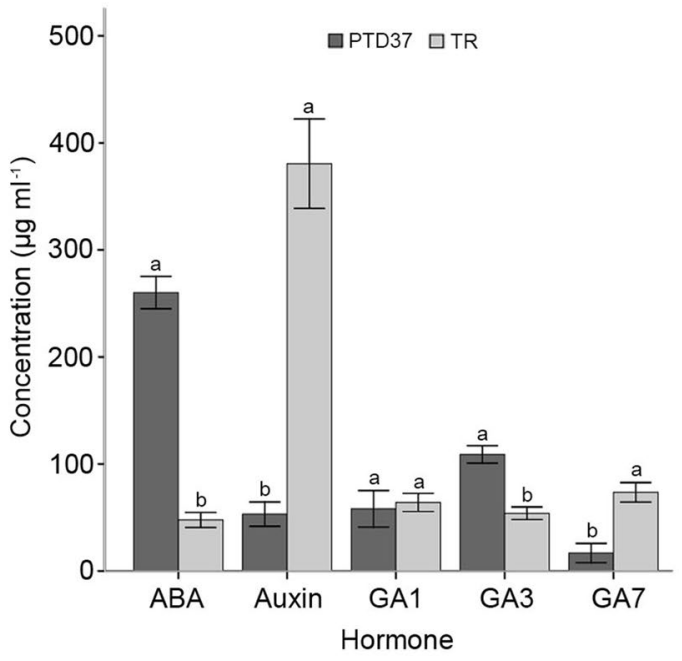

b

Figure 5. Effect of PEG application on the leaf ABA content of seedlings under different treatments and the levels of different hormones in PTD37 and T. harzianum fermentation broth. (a) Effect of PEG application on the leaf ABA content of seedlings under different treatments. (b) Levels of ABA, auxin, GA1, GA3 and GA7 in PTD37 and T. harzianum fermentation broth. PDB = potato dextrose broth; PTD37 fermentation broth supernatant of the PTD37 strain, TR fermentation broth supernatant of T. harzianum. Data are the means \pm SD from five independent tests. Different letters on error bars indicate significant differences at $P=0.05$ between the ABA contents of seedlings from different treatments on the same day after PEG application. They also indicate significant differences at $P=0.05$ between the analyzed hormone levels of different fungal fermentation broths. The values on day 0 after PEG treatment indicate that they were measured before PEG application.

and molecular data, the current isolate was identified as a species of the genus Phoma. In the past, no species improving plant stress resistance was found in this genus. The species in the genus Phoma are generally pathogenic fungi and often cause decay or wilting in the host plant ${ }^{34,35}$. Our findings may partly support a hypothesis that the harmful parasitic relationship between specific pathogenic microorganisms and their host plants can become mutually beneficial relationships through coordinated evolution ${ }^{36,37}$.

The current study revealed that the PTD37-treated P. tabulaeformis seedlings had significantly higher antioxidant activity than other treatments. Plants quickly accumulate reactive oxygen species under abiotic stresses, which are often scavenged by the antioxidants they produce to prevent damage to their tissues and organs ${ }^{38-40}$. 
Some researchers have demonstrated that symbiotic endophytes can promote the production of antioxidant compounds in plants to improve plant abiotic stress tolerance ${ }^{21,41,42}$. Piriformospora indica was also found to upregulate antioxidant activities in inoculated plants to enhance salt ${ }^{43}$ and drought resistance ${ }^{44}$. These reports are consistent with our finding that PTD37-treated seedlings showed higher drought tolerance than seedlings in other treatments. It is proposed that antioxidant activities may be a major mechanism of improvement of drought resistance in P. tabulaeformis by PTD37 FBS.

Our study showed that the chlorophyll, water, and MDA contents in the PTD37-treated seedlings were altered less than those in the other treatments after PEG application. Drought causes plant chlorophyll degradation, MDA accumulation, and water loss in tissues, which result in a decline in photosynthesis ${ }^{45-47}$. However, drought-tolerant plants can reduce chlorophyll degradation, MDA accumulation, and water loss compared with drought-sensitive plants. Plants inoculated with certain endophytes can maintain higher water and chlorophyll contents and lower MDA levels than noninoculated plants under drought stress, suggesting that these microbes can enhance plant drought resistance ${ }^{16,17,22,23,28-30}$. Similar to the above reports, $P$. tabulaeformis seedlings treated with PTD37 accumulated less MDA, broke down less chlorophyll, and lost less tissue water than the plants in other treatments. This result indicated that PTD37 may play a role in the improvement of chlorophyll, MDA, and water contents under drought stress.

The PTD37 fermentation broth contained high levels of ABA in the present study. ABA is considered a crucial signaling substance for plant stress-tolerance signaling pathways ${ }^{48-50}$. Drought can trigger ABA formation, causing stomatal closure and decreasing photosynthesis ${ }^{51-54}$. It has been demonstrated that application of exogenous ABA can induce a plant's drought tolerance by increasing enzymatic and nonenzymatic antioxidant production ${ }^{55-57}$. The application of broth containing ABA secreted by PTD37 yielded similar results to that of exogenous ABA. Plants treated with the PTD37 fermentation broth showed a higher drought tolerance than plants in other treatments. Therefore, ABA in PTD37 fermentation broth may trigger a series of drought-resistance responses in seedlings to enhance drought resistance.

A primary response of plants under drought stress is proline accumulation, which weakens the damage caused to tissues and cells ${ }^{30,45}$. Proline accumulation is considered to protect membranes and proteins from damage by reactive oxygen species and to play a role in osmotic adjustment under abiotic stress ${ }^{28,30,45}$. Our study showed that the seedlings in the PTD37 treatment had a higher proline content than those in the other treatments. Some researchers have shown that plants inoculated with endophytes can produce less proline than noninoculated plants under drought conditions ${ }^{16,23}$. However, more researchers have demonstrated that inoculated plants have higher levels of proline than noninoculated plants under drought conditions ${ }^{23,24,28,29,46}$. The results of the current study are consistent with the conclusions of most published literature. After PEG application, PTD37-treated seedlings were more drought resistant than other seedlings. This may suggest that the enhanced proline production in PTD37-treated seedlings may reduce the damage to cells caused by drought.

Endophytes can enhance the ability of plants to resist drought by increasing plant total biomass, aboveground or belowground biomass, tiller number, root length, and root number ${ }^{14-20}$. In this study, the basal diameter, height, and total biomass of PTD37-treated seedlings were not significantly higher than those of other treatments. This may imply that PTD37 does not strengthen a seedling's drought tolerance through biomass, height, and basal diameter. However, we did not measure the aboveground and belowground biomass, so it is not clear whether PTD37 affects biomass allocation to improve seedling drought tolerance.

In the current research, a potential endophytic fungus enhancing $P$. tabulaeformis drought tolerance was isolated, and the mechanism by which FBS enhances the drought resistance of seedlings was studied. This will facilitate the development of an alternative to traditional methods for drought resistance of $P$. tabulaeformis and will provide a better understanding of the mechanism by which fungal endophytes improve plant drought resistance. However, there is still much work to be done in the future. Potential endophytes should be inoculated into P. tabulaeformis, and the mechanism by which endophytes strengthen plant drought resistance requires further research. The molecular basis of the relationship between the endophyte and plants also needs to be studied.

\section{Materials and methods}

Materials. This experimental study complies with Chinese national and local laws, and sample collection has been permitted by the local relevant administration. The root samples used to isolate the endophytes were collected from three mature Chinese pine trees grown on the top of a south-facing arid cliff $1300 \mathrm{~m}$ above sea level in the Taihang Mountains. Each tree provided 16 root segments (20-30 mm long, 3-5 mm diameter), which were taken from roots at different positions. After collection, the samples were immediately rinsed with distilled water, placed in a sealed fresh-keeping bag, and stored in a refrigerator at $4{ }^{\circ} \mathrm{C}$. Voucher specimens (ID: TH2017022101-6) for the trees were deposited at the Herbarium of Henan Institute of Science and Technology and were identified by Dr. Pengming Yang.

Reagents. Analytical-grade reagents were used to determine the POD, CAT, SOD, MDA, proline, and chlorophyll levels (Sangon Biotech [Shanghai] Co., Ltd. or Solarbio Science and Technology [Shanghai] Co., Ltd.). All deuterated standards and calibration samples for determining the ABA, auxin, GA1, GA3, and GA7 levels were purchased from Sigma-Aldrich (St. Louis, MO, USA). Reagents for the high-performance liquid phase were sourced from Thermo Fisher Scientific Inc. Consumables such as petri dishes, agar, and sucrose for endophytic isolation and fermentation were purchased from Solarbio Science and Technology Co., Ltd. PDA, PDB, or water agar (WA) media were used. The PDB medium contained $200 \mathrm{~g} \mathrm{l}^{-1}$ potato and $20 \mathrm{~g} \mathrm{l}^{-1} \mathrm{D}$-glucose. The PDA medium contained $200 \mathrm{~g} \mathrm{l}^{-1}$ potato, $20 \mathrm{~g} \mathrm{l}^{-1} \mathrm{D}$-glucose and $15 \mathrm{~g} \mathrm{l}^{-1}$ agar. The WA medium was prepared by adding $10 \mathrm{~g}$ agar to $1000 \mathrm{ml}$ distilled water. 
Endophytic isolation. The Chinese pine root samples were thoroughly rinsed with sterilized distilled water, and their surfaces were washed with a clean brush to remove the soil particles and other impurities on the roots. The surface-cleaned roots were sterilized in $75 \%$ alcohol for $4 \mathrm{~min}$ and then cut into small pieces ( $\sim \mathrm{mm} \mathrm{long})$. The pieces were sterilized in $0.1 \%$ mercury chloride solution for $5 \mathrm{~min}$ and then rinsed with sterile distilled water for $3 \mathrm{~min}$ to remove residual mercuric chloride, as per Li et al. $(2015)^{58}$. The sterilized root pieces were then placed on $1.0 \%$ WA medium and cultivated in the dark at $22-23^{\circ} \mathrm{C}$. Three blank controls containing only WA were cultivated under the same conditions. All cultivars were carefully observed daily. After 3-5 days, white mycelia or hyphae of the endophytes emerged on the root piece surfaces. These hyphae were transferred to PDA medium for further cultivation. Hyphae that arose on the newly positioned root pieces were isolated and cultured to obtain as many different endophytic strains as possible. After 1.5 months, most endophytes growing on the WA medium were isolated. Primary isolates were further purified to ensure a single strain in each medium.

Preparation of the FBS of the isolates and T. harzianum. Each isolate was inoculated in $200 \mathrm{ml}$ PDB medium in a triangular flask and then cultured in a 150 -rpm shaker at $25^{\circ} \mathrm{C}$ for 10 days. Afterward, this fermentation broth was centrifuged at $7000 \times g$ for $30 \mathrm{~min}$, and $100 \mathrm{ml}$ of supernatant was taken for the following tests. T. Harzianum was fermented under the same conditions, and its FBS was obtained. T. harzianum and other Trichoderma spp. can enhance abiotic and biotic stress resistance in plants ${ }^{59-62}$.

Screening of the isolates enhancing the drought-stress tolerance of Chinese pine seedlings. Chinese pine seeds were disinfected, soaked and germinated. After radicles emerged from $\sim 80 \%$ of the seeds, every 10 germinated seeds were sown in a square plastic plant pot $(12 \mathrm{~cm}$ wide, $10 \mathrm{~mm}$ high) containing a sterile mixture of river sand and perlite $(2: 1 \mathrm{v} / \mathrm{v})$ that was autoclaved at $125^{\circ} \mathrm{C}$ for $45 \mathrm{~min}$. Next, 562 pots were prepared, numbered, and transferred to a greenhouse at $23-25^{\circ} \mathrm{C}$ with $60-70 \%$ relative humidity. When all seedlings emerged, they were watered with $2 \mathrm{ml} /$ plant of Hogland's nutrient solution every 5 days and were sprayed with distilled water to prevent drought. After $\sim 20$ days, when the seedlings grew approximately 20 needles, 279 treatments were completely randomly assigned to 558 of the 562 pots. The treatments comprised three FBS dilutions (twofold, fivefold, and tenfold) of each of the 93 isolates, with two replicates per treatment. The remaining 4 pots were used as controls and were watered with $3 \mathrm{ml}$ distilled water per plant every 3 days. The other seedlings were treated with $3 \mathrm{ml}$ diluted FBS from each relevant isolate per plant every 3 days. After 21 days, each pot was treated with $50 \mathrm{ml}$ 25\% PEG6000 solution except that 2 of 4 control pots were treated with $50 \mathrm{ml}$ distilled water. After 15 days, the plants with enhanced drought tolerance were differentiated based on their morphological characteristics, such as leaf color and wilting degree, and the strain that best promoted drought resistance in Chinese pine was determined.

Experimental design of the effect of the target fungus (PTD37 strain) on Chinese pine seedling physiological and biochemical characteristics. Chinese pine seeds were sterilized, germinated, and planted in pots, as described above. Then, 120 pots of seedlings with $\sim 20$ needles were prepared and randomly combined into 20 experimental units, each consisting of 6 completely randomly selected pots. Five pots were then independently assigned to each of four treatments as follows: application of $3 \mathrm{ml}$ fivefold PTD37 FBS per plant, application of $3 \mathrm{ml}$ fivefold T. harzianum FBS per plant, application of fivefold PDB medium FBS per plant, and application of $3 \mathrm{ml}$ distilled water per plant. Afterward, seedlings from each experimental unit were treated with $3 \mathrm{ml}$ fivefold FBS or distilled water per plant every 3 days according to the experimental design. After 21 days, all seedlings were treated with $5 \mathrm{ml}$ 25\% PEG6000 solution per plant.

Measurement of the seedling basal diameter and height. Before application of PEG6000 solution, the seedling basal diameters and heights were measured to evaluate the different treatment effects. Three plants from each treatment were selected randomly and measured with a digital Vernier caliper. This measurement was repeated three times.

Determination of the total fresh biomass, dry biomass, and leaf water content. Before application of PEG6000 solution, three plants per treatment were randomly selected to measure the total fresh biomass using a 0.0001-mg electronic analytical balance. This measurement was repeated three times. The plants were similarly sampled again, oven-dried at $70{ }^{\circ} \mathrm{C}$ for $72 \mathrm{~h}$ and weighed. After PEG application, plants of different treatments were sampled every 3 days. Three seedlings were randomly sampled and weighed immediately; this was repeated three times. The samples were oven-dried at $70^{\circ} \mathrm{C}$ for $72 \mathrm{~h}$ and weighed. The leaf water content was determined based on the leaf fresh and dry weights.

Leaf sample collection to determine the physiological and biochemical characteristics of the seedlings. After treatment for 21 days, the heights and basal diameters of 8 randomly selected plants per replication were measured. The needles of 8 randomly selected plants per replication were collected and immediately stored in a refrigerator at $-80^{\circ} \mathrm{C}$ to determine the antioxidant compound and enzyme activity levels. On days 1, 3, 6, and 9 after PEG treatment, the leaf samples were collected and stored to determine the compound levels and enzyme activities as described above.

Estimation of SOD, POD, and CAT activities in Chinese pine seedling leaves. SOD, POD, and catalase activities were determined according to Sunkar $(2010)^{59}$ and Zhang $(2011)^{60}$. 
Estimation of SOD activity. Chinese pine leaves $(0.2 \mathrm{~g})$ stored at $-80^{\circ} \mathrm{C}$ were added to a mortar containing $2 \mathrm{ml}$ of $0.05 \mathrm{M}$ phosphate-buffered saline (PBS: $\mathrm{pH} 7.8$ ) in an ice bath and quickly ground. Then, $8 \mathrm{ml}$ PBS was transferred into this mortar to obtain a $10-\mathrm{ml}$ final volume. Next, $5 \mathrm{ml}$ of ground leaf tissue mixture was transferred into a 5 -ml centrifuge tube and centrifuged at $12,000 \mathrm{rpm}$ for $10 \mathrm{~min}$, and then $50 \mu \mathrm{l}$ of supernatant was placed in a new centrifuge tube. Next, $300 \mu \mathrm{l} 130 \mu \mathrm{M}$ methionine, $300 \mu \mathrm{l} 750 \mu \mathrm{M}$ nitrotetrazolium blue chloride, $300 \mu \mathrm{l}$ $100 \mu \mathrm{M}$ EDTA-Na2 solution, $300 \mu \mathrm{l} 20 \mu \mathrm{M}$ riboflavin solution, and $250 \mu \mathrm{l}$ distilled water were added to this tube in sequence to obtain a $3-\mathrm{ml}$ final volume. The test tube was placed under a $4000 \mathrm{l} \times$ fluorescent lamp at $24{ }^{\circ} \mathrm{C}$ for $20 \mathrm{~min}$; an enzyme-free reaction system used as the control. The absorbance of this system was measured at $560 \mathrm{~nm}$.

Estimation of POD activity. Chinese pine leaves $(0.2 \mathrm{~g})$ stored at $-80{ }^{\circ} \mathrm{C}$ were placed in a mortar with $2 \mathrm{ml}$ of $100 \mathrm{mM}$ PBS buffer (pH 6.0) in an ice bath and quickly ground. Then, $8 \mathrm{ml}$ PBS was added to the mortar to obtain a $10 \mathrm{ml}$ final volume. The ground tissue mixture $(5 \mathrm{ml})$ was transferred to a $5-\mathrm{ml}$ centrifuge tube and centrifuged at $12,000 \mathrm{rpm}$ for $10 \mathrm{~min}$. The supernatant was transferred into a 20 -ml volumetric flask and brought to a total volume of $20 \mathrm{ml}$ with PBS buffer ( $\mathrm{pH}$ 6.0). Next, $0.1 \mathrm{ml}$ of the sample solution was transferred into a test tube containing $2.3 \mathrm{ml}$ of PBS, $0.5 \mathrm{ml} 5 \mathrm{mM}$ guaiacol and $0.1 \mathrm{ml} 4 \mathrm{mM}$ hydrogen peroxide solution. The absorbance of the reaction system was measured every $30 \mathrm{~s}$ at $470 \mathrm{~nm}$. A reaction system containing no sample was used as a control.

Estimation of CAT activity. Chinese pine leaves $(0.2 \mathrm{~g})$ stored at $-80^{\circ} \mathrm{C}$ were ground in a mortar containing $2 \mathrm{ml} 0.05 \mathrm{M}$ PBS (pH 7.0) in an ice bath and $8 \mathrm{ml}$ PBS was added to obtain a 10-ml final volume. Next, $5 \mathrm{ml}$ of sample solution was added to a $5-\mathrm{ml}$ centrifuge tube and centrifuged at $5000 \mathrm{rpm}$ in a refrigerated centrifuge for 20 min. Afterward, $0.2 \mathrm{ml}$ of supernatant was transferred into a test tube and mixed with $1.5 \mathrm{ml}$ PBS and $1 \mathrm{ml}$ distilled water. Next, $0.3 \mathrm{ml}$ of $0.1 \mathrm{M}$ hydrogen peroxide solution was added to this tube. The absorbance of the reaction system was measured immediately at $240 \mathrm{~nm}$ and then every minute for $4 \mathrm{~min}$. Enzyme-denatured reaction systems were used as controls.

Valuation of MDA, proline, and chlorophyll contents in Chinese pine seedling leaves. The MDA, proline, and chlorophyll contents were determined following Sunkar $(2010)^{63}$ and Zhang $(2011)^{64}$ with slight modifications.

Measurement of the MDA content. Chinese pine leaves $(0.2 \mathrm{~g})$ stored at $-80{ }^{\circ} \mathrm{C}$ and $2 \mathrm{ml} 10 \%$ trichloroacetic acid (TCA) were mixed and ground in a mortar in an ice bath, and $8 \mathrm{ml}$ of $10 \%$ TCA was added to the mortar to obtain a 10-ml final volume. Next, $5 \mathrm{ml}$ of sample solution was transferred to an Eppendorf tube and centrifuged at $5000 \mathrm{rpm}$ in a refrigerated centrifuge for $20 \mathrm{~min}$. Then, $1 \mathrm{ml}$ of the supernatant was taken and mixed with $3 \mathrm{ml} 0.6 \% 2$-thiobarbituric acid. The mixed solution was heated in a boiling bath for $20 \mathrm{~min}$, rapidly cooled in an ice bath, and centrifuged at $1000 \mathrm{rpm}$ for $5 \mathrm{~min}$. Afterward, the supernatant was taken, and its absorbance was estimated at 532, 600, and $450 \mathrm{~nm}$.

Measurement of the proline content. Chinese pine leaves $(0.2 \mathrm{~g})$ stored at $-80^{\circ} \mathrm{C}$ were ground to a slurry in a mortar with $2 \mathrm{ml}$ of $3 \% 5$-sulfosalicylic acid dehydrate, and then $8 \mathrm{ml} 3 \% 5$-sulfosalicylic acid dehydrate was transferred into this mortar. The sample solution $(5 \mathrm{ml})$ was added to a centrifuge tube, and then heated and shaken in a boiling water bath for $10 \mathrm{~min}$. The solution was then cooled in a $20^{\circ} \mathrm{C}$ water bath and centrifuged at $5000 \mathrm{rpm}$ for $10 \mathrm{~min}$. Then, $1 \mathrm{ml}$ of the supernatant was transferred to a $10-\mathrm{ml}$ test tube with a lid. Next, $2 \mathrm{ml}$ glacial acetic acid and $2 \mathrm{ml}$ acid ninhydrin were added to this tube, which was sealed with a lid to prevent evaporation, and then heated in boiling water for $30 \mathrm{~min}$. After cooling in a $20^{\circ} \mathrm{C}$ water bath, the solution was mixed with $4 \mathrm{ml}$ toluene, shaken for $30 \mathrm{~s}$, and then left to stand for $2 \mathrm{~h}$. Finally, $0.5 \mathrm{ml}$ of the red proline toluene solution, located at the top of the system, was carefully removed, and its absorbance was read at $520 \mathrm{~nm}$.

Measurement of the chlorophyll content. Chinese pine leaves $(0.2 \mathrm{~g})$ stored at $-80^{\circ} \mathrm{C}$ were ground in a mortar containing $2 \mathrm{ml}$ ethanol, and then $8 \mathrm{ml}$ ethanol was added. The sample in the mortar was transferred into a $15-\mathrm{ml}$ plastic test tube, and then the sample solution was fully oscillated on a vortex oscillator and filtered. The filtrate was brought to a 50-ml total volume with ethanol, and its absorbance was measured at 710, 665 (chlorophyll $a$ ), and $649 \mathrm{~nm}$ (chlorophyll $b$ ). The extinction coefficients of chlorophyll $a$ and $b$ were $72.23 \mathrm{l} \mathrm{g}^{-1} \mathrm{~cm}^{-1}$ and $38.52 \mathrm{~g}^{-1} \mathrm{~cm}^{-1}$, respectively, when the spectrophotometer was zeroed at $710 \mathrm{~nm}$.

Detection of the hormone content. Hormone levels in the fungal fermentation broth and Chinese pine leaves were determined as per Pan et al. $(2010)^{65}$.

Detection of ABA, auxin, GA1, GA3, and GA7 levels in endophytes and T. harzianum fermentation broth. Fermentation broth $(5 \mathrm{ml})$ was centrifuged at $6000 \mathrm{rpm}$ for $10 \mathrm{~min} ; 1.9 \mathrm{ml}$ of supernatant was added to a $5-\mathrm{ml}$ centrifuge tube, and then $100 \mu \mathrm{l}$ deuterated hormone internal standard and $2 \mathrm{ml}$ dichloromethane were added. The tube was shaken on a constant temperature shaker oscillator at $4{ }^{\circ} \mathrm{C}$ for $30 \mathrm{~min}$. The mixed solution was centrifuged at 12,000 rpm at $4{ }^{\circ} \mathrm{C}$ for $5 \mathrm{~min}$. The bottom layer solution $(900 \mu \mathrm{l})$ was taken, transferred to a $2-\mathrm{ml}$ centrifuge tube and evaporated in a nitrogen evaporator until a light-brown wet cream formed. The cream was dissolved in $200 \mu \mathrm{l}$ methanol. Finally, $25 \mu \mathrm{l}$ of sample solution was injected into a C18 Gemini column (Phenomenex USA) for HPLC-ESI-MS/MS analysis. 
Detection of $A B A$ contents in seedlings leaves. Chinese pine leaves $(0.2 \mathrm{~g})$ stored at $-80{ }^{\circ} \mathrm{C}$ were ground into powder in liquid nitrogen in a mortar, and then $100 \mathrm{mg}$ of the powder was transferred into a 5-ml centrifuge tube. Next, $100 \mu \mathrm{l}$ ABA deuterated internal standard and $1 \mathrm{ml}$ extraction solution were added to the tube and incubated in a shaker at $100 \mathrm{rpm}$ at $4^{\circ} \mathrm{C}$ for $30 \mathrm{~min}$. Afterward, $2 \mathrm{ml}$ dichloromethane was added to the solution, shaken on a constant temperature shaker oscillator at $4^{\circ} \mathrm{C}$ for $30 \mathrm{~min}$, and then centrifuged at $12,000 \mathrm{rpm}$ at $4{ }^{\circ} \mathrm{C}$ for $5 \mathrm{~min}$. Next, $900 \mu \mathrm{l}$ of solution from the lower layer was transferred to a 2-ml Eppendorf tube and evaporated to form a wet cream in a nitrogen evaporator. The cream was mixed with $200 \mu \mathrm{l}$ methanol and dissolved. Finally, $25 \mu \mathrm{l}$ of sample solution was injected into a C18 Gemini column (Phenomenex USA) for HPLC-ESI-MS/MS analysis.

Morphological and molecular identification of the endophytic fungi. After culturing the isolates in PDA medium at $27-28{ }^{\circ} \mathrm{C}$ for $7-15$ days, the hyphae, mycelium, conidia, and conidiophores were observed and photographed with an optical microscope. Endophytic DNA was extracted as per Raeder and Broda $(1985)^{66}$. The ITS (ITS1 5'-TCCGTAGGTGAACCTGCGG-3'; ITS2 5'-TCCTCCGCTTATTGATATGC-3') and 18S (NS1 5'-GTAGTCATATGCTTGTCTC-3'; NS2 5'-GGCTGCTGGCACCAGACTTGC-3') regions were amplified by PCR and sequenced as per Simon et al. (1992) ${ }^{67}$. The ITS and $18 \mathrm{~S}$ regions were compared against available known sequences in GenBank using BLAST and their classified statuses were determined based on the molecular and morphological data.

Statistical analysis. Normality and variance constancy were determined for all data. All data were normally distributed. One-way analysis of variance and least significant difference multiple comparison tests were used to determine differences between the analyzed traits of the seedlings in different treatments. Independent t-tests were used to test differences in hormone contents between the PTD37 strain and T. harzianum fermentation broth. P-values $<0.05$ were considered statistically significant. Compound bar creation and statistical analyses were performed using SPSS 17.0 for Windows (SPSS, Inc., Chicago, IL, USA).

Received: 15 February 2020; Accepted: 15 March 2021

Published online: 07 April 2021

\section{References}

1. Williams, A. P. et al. Temperature as a potent driver of regional forest drought stress and tree mortality. Nat. Clim. Change 3, 292-297 (2013).

2. Allen, C. D., Breshears, D. D. \& McDowell, N. G. On underestimation of global vulnerability to tree mortality and forest die-off from hotter drought in the Anthropocene. Ecosphere 6, 1-55 (2015).

3. Farooq, M., Wahid, A., Kobayashi, N., Fujita, D. \& Basra, S. M. A. Plant drought stress: Effects, mechanisms and management. In Sustainable Agriculture (eds Lichtfouse, E. et al.) 153-188 (Springer, 2009).

4. Fang, Y. \& Xiong, L. General mechanisms of drought response and their application in drought resistance improvement in plants. Cell. Mol. Life Sci. 72, 673-689 (2015).

5. Basu, S., Ramegowda, V., Kumar, A. \& Pereira, A. Plant adaptation to drought stress. F1000Research 5, 1554. https://doi.org/10. 12688/f1000research.7678.1 (2016).

6. Fathi, A. \& Tari, D. B. Effect of drought stress and its mechanism in plants. Int. J Life Sci. 10, 1-6 (2016).

7. Nakashima, K., Ito, Y. \& Yamaguchi-Shinozaki, K. Transcriptional regulatory networks in response to abiotic stresses in Arabidopsis and grasses. Plant Physiol. 149, 88-95 (2009).

8. Peleg, Z. \& Blumwald, E. Hormone balance and abiotic stress tolerance in crop plants. Curr. Opin. Plant Biol. 14, 290-295 (2011).

9. Newcombe, G. Endophytes in forest management: four challenges. In Endophytes of Forest Trees (eds Pirttilä, A. M. \& Frank, A. C.) 251-262 (Springer, 2011)

10. Rodriguez, R. J., Redman, R. S. \& Henson, J. M. The role of fungal symbioses in the adaptation of plants to high stress environments. Mitig. Adapt. Strateg. Glob. Change 9, 261-272 (2004).

11. Vandenkoornhuyse, P., Quaiser, A., Duhamel, M., Le Van, A. \& Dufresne, A. The importance of the microbiome of the plant holobiont. New Phytol. 206, 1196-1206 (2015).

12. Lau, J. A., Lennon, J. T. \& Heath, K. D. Trees harness the power of microbes to survive climate change. Proc. Natl. Acad. Sci. 114, 11009-11011 (2017).

13. Lau, J. A. \& Lennon, J. T. Rapid responses of soil microorganisms improve plant fitness in novel environments. Proc. Natl. Acad. Sci. 109, 14058-14062 (2012).

14. González-Teuber, M., Urzúa, A., Plaza, P. \& Bascuñán-Godoy, L. Effects of root endophytic fungi on response of Chenopodium quinoa to drought stress. Plant Ecol. 219, 231-240 (2018).

15. Li, X. et al. Dark septate endophytes improve the growth of host and non-host plants under drought stress through altered root development. Plant Soil 439, 259-272 (2019).

16. Ferus, P., Barta, M. \& Konôpková, J. Endophytic fungus Beauveria bassiana can enhance drought tolerance in red oak seedlings. Trees 33, 1179-1186 (2019).

17. Naveed, M., Mitter, B., Reichenauer, T. G., Wieczorek, K. \& Sessitsch, A. Increased drought stress resilience of maize through endophytic colonization by Burkholderia phytofirmans PsJN and Enterobacter sp. FD17. Environ. Exp. Bot. 97, 30-39 (2014).

18. Kane, K. H. Effects of endophyte infection on drought stress tolerance of Lolium perenne accessions from the Mediterranean region. Environ. Exp. Bot. 71, 337-344 (2011).

19. Ren, A. et al. Benefits of a fungal endophyte in Leymus chinensis depend more on water than on nutrient availability. Environ. Exp. Bot. 108, 71-78 (2014).

20. Murphy, B. R., Martin Nieto, L., Doohan, F. M. \& Hodkinson, T. R. Fungal endophytes enhance agronomically important traits in severely drought-stressed barley. J. Agron. Crop Sci. 201, 419-427 (2015).

21. Khan, Z. et al. Growth enhancement and drought tolerance of hybrid poplar upon inoculation with endophyte consortia. Curr. Plant Biol. 6, 38-47 (2016).

22. Zhang, Q. et al. Dark septate endophyte improves drought tolerance in Sorghum. Int. J. Agric. Biol. 19, 53-60 (2017).

23. Naveed, M., Hussain, M. B., Zahir, Z. A., Mitter, B. \& Sessitsch, A. Drought stress amelioration in wheat through inoculation with Burkholderia phytofirmans strain PsIN. Plant Growth Regul. 73, 121-131 (2014).

24. Ghaffari, M. R. et al. Root endophytic fungus Piriformospora indica improves drought stress adaptation in barley by metabolic and proteomic reprogramming. Environ. Exp. Bot. 157, 197-210 (2019). 
25. Forni, C., Duca, D. \& Glick, B. R. Mechanisms of plant response to salt and drought stress and their alteration by rhizobacteria. Plant Soil 410, 335-356 (2017).

26. Cohen, A. C. et al. Azospirillum brasilense ameliorates the response of Arabidopsis thaliana to drought mainly via enhancement of ABA levels. Physiol. Plant 153, 79-90 (2015).

27. Ruiz-Lozano, J. M. et al. Arbuscular mycorrhizal symbiosis induces strigolactone biosynthesis under drought and improves drought tolerance in lettuce and tomato. Plant Cell Environ. 39, 441-452 (2016).

28. Ullah, A. et al. Drought tolerance improvement in plants: an endophytic bacterial approach. Appl. Microbiol. Biotechnol. 103, 7385-7397 (2019).

29. Nagabhyru, P., Dinkins, R. D., Wood, C. L., Bacon, C. W. \& Schardl, C. L. Tall fescue endophyte effects on tolerance to water-deficit stress. BMC Plant Boil. 13, 127. https://doi.org/10.1186/1471-2229-13-127 (2013).

30. He, L., Hatier, J. H. \& Matthew, C. Drought tolerance of two perennial ryegrass cultivars with and without AR37 endophyte. N. Z. J. Agric. Res. 60, 173-188 (2017).

31. Hahn, H. et al. Neotyphodium fungal endophytes confer physiological protection to perennial ryegrass (Loliumperenne L.) subjected to a water deficit. Environ. Exp. Bot. 63, 183-199 (2008).

32. Aveskamp, M. M., De Gruyter, J. \& Crous, P. W. Biology and recent developments in the systematics of Phoma, a complex genus of major quarantine significance. Fungal Divers. 31, 1-18 (2008).

33. Aveskamp, M. M., De Gruyter, J., Woudenberg, J. H. C., Verkley, G. J. M. \& Crous, P. W. Highlights of the Didymellaceae: A polyphasic approach to characterise Phoma and related pleosporalean genera. Stud. Mycol. 65, 1-60 (2010).

34. Rodriguez, R., Leath, K. T. \& Hill, R. R. Pathogenicity of Phomamedicaginis var. medicaginis to roots of alfalfa. Plant Dis. 74, 680-683 (1990).

35. Bennett, A., Ponder, M. M. \& Garcia-Diaz, J. Phoma infections: Classification, potential food sources, and their clinical impact. Microorganisms 6, 58 (2018).

36. Carroll, G. Fungal endophytes in stems and leaves: from latent pathogen to mutualistic symbiont. Ecology 69, 2-9 (1988).

37. Hardoim, P. R. et al. The hidden world within plants: Ecological and evolutionary considerations for defining functioning of microbial endophytes. Microbiol. Mol. Biol. Rev. 79, 293-320 (2015).

38. Reddy, A. R., Chaitanya, K. V. \& Vivekanandan, M. Drought-induced responses of photosynthesis and antioxidant metabolism in higher plants. J. Plant Physiol. 161, 1189-1202 (2004).

39. Ahmad, P., Jaleel, C. A., Salem, M. A., Nabi, G. \& Sharma, S. Roles of enzymatic and nonenzymatic antioxidants in plants during abiotic stress. Crit. Rev. Biotechnol. 30, 161-175 (2010).

40. Hasanuzzaman, M., Hossain, M. A., da Silva, J. A. T. \& Fujita, M. Plant response and tolerance to abiotic oxidative stress: Antioxidant defense is a key factor. In Crop stress and its management: Perspectives and strategies (eds Venkateswarlu, B. et al.) 261-315 (Springer, 2012).

41. White, J. F. Jr. \& Torres, M. S. Is plant endophyte-mediated defensive mutualism the result of oxidative stress protection?. Physiol. Plant. 138, 440-446 (2010).

42. Hamilton, C. E. \& Bauerle, T. L. A new currency for mutualism? Fungal endophytes alter antioxidant activity in hosts responding to drought. Fungal Divers. 54, 39-49 (2012).

43. Baltruschat, H. et al. Salt tolerance of barley induced by the root endophyte Piriformospora indica is associated with a strong increase in antioxidants. New Phytol. 180, 501-510 (2008).

44. Sun, C. et al. Piriformospora indica confers drought tolerance in Chinese cabbage leaves by stimulating antioxidant enzymes, the expression of drought-related genes and the plastid-localized CAS protein. J. Plant Physiol. 167, 1009-1017 (2010).

45. Anjum, S. A. et al. Morphological, physiological and biochemical responses of plants to drought stress. Afr. J Agric. Res. 6, 2026$2032(2011)$.

46. Fathi, A. \& Tari, D. B. Effect of drought stress and its mechanism in plants. Int. J. Life Sci. 10, 1-6 (2016).

47. Xiang, J. et al. Overexpressing heat-shock protein OsHSP50. 2 improves drought tolerance in rice. Plant Cell Rep. 37, 1585-1595 (2018).

48. Lee, S. C. \& Luan, S. ABA signal transduction at the crossroad of biotic and abiotic stress responses. Plant Cell Environ. 35, 53-60 (2012).

49. Kim, T. H. Mechanism of ABA signal transduction: agricultural highlights for improving drought tolerance. J. Plant Biol. 57, 1-8 (2014).

50. Vishwakarma, K. et al. Abscisic acid signaling and abiotic stress tolerance in plants: a review on current knowledge and future prospects. Front. Plant Sci. 8, 161 (2017).

51. Seki, M., Umezawa, T., Urano, K. \& Shinozaki, K. Regulatory metabolic networks in drought stress responses. Curr. Opin. Plant Biol. 10, 296-302 (2007).

52. Leung, J. \& Giraudat, J. Abscisic acid signal transduction. Annu. Rev. Plant Biol. 49, 199-222 (1998).

53. Finkelstein, R. R., Gampala, S. S. \& Rock, C. D. Abscisic acid signaling in seeds and seedlings. Plant Cell 14(suppl. 1), S15-S45 (2002).

54. Downton, W. J. S., Loveys, B. R. \& Grant, W. J. R. Stomatal closure fully accounts for the inhibition of photosynthesis by abscisic acid. New Phytol. 108, 263-266 (1988).

55. Ibrahim, M. H. \& Jaafar, H. Z. Abscisic acid induced changes in production of primary and secondary metabolites, photosynthetic capacity, antioxidant capability, antioxidant enzymes and lipoxygenase inhibitory activity of Orthosiphon stamineus Benth. Molecules 18, 7957-7976 (2013).

56. Teng, K. et al. Exogenous ABA induces drought tolerance in upland rice: the role of chloroplast and ABA biosynthesis-related gene expression on photosystem II during PEG stress. Acta Physiol. Plant. 36, 2219-2227 (2014).

57. Gai, Z. et al. Exogenous abscisic acid induces the lipid and flavonoid metabolism of tea plants under drought stress. Sci. Rep. 10, 1-13 (2020).

58. Li, Y., Yang, J., Zhou, X., Zhao, W. \& Jian, Z. Isolation and identification of a 10-deacetyl baccatin-III-producing endophyte from Taxus wallichiana. Appl. Biochem. Biotechnol. 175, 2224-2231 (2015).

59. Rawat, L., Singh, Y., Shukla, N. \& Kumar, J. Alleviation of the adverse effects of salinity stress in wheat (Triticumaestivum L.) by seed biopriming with salinity tolerant isolates of Trichodermaharzianum. Plant Soil 347, 387-400 (2011).

60. Mastouri, F., Björkman, T. \& Harman, G. E. Trichoderma harzianum enhances antioxidant defense of tomato seedlings and resistance to water deficit. Mol. Plant Microbe Interact. 25, 1264-1271 (2012).

61. Zaidi, N. W., Dar, M. H., Singh, S. \& Singh, U. S. Trichoderma species as abiotic stress relievers in plants. In Biotechnology and Biology of Trichoderma (eds Gupta, V. G. et al.) 515-525 (Elsevier, 2014).

62. Pandey, V. et al. Dose-dependent response of Trichoderma harzianum in improving drought tolerance in rice genotypes. Planta 243, 1251-1264 (2016).

63. Sunkar, R. (ed.) Plant Stress Tolerance: Methods and Protocols (Humana, 2010).

64. Zhang, S. Q. (ed.) Plant Physiology Experimental Techniques Tutorial (Science, 2011).

65. Pan, X., Welti, R. \& Wang, X. Quantitative analysis of major plant hormones in crude plant extracts by high-performance liquid chromatography-mass spectrometry. Nat. Protoc. 5, 986-992 (2010).

66. Raeder, U. \& Broda, P. Rapid preparation of DNA from filamentous fungi. Lett. Appl. Microbiol. 1, 17-20 (1985). 
67. Simon, L., Lalonde, M. \& Bruns, T. D. Specific amplification of 18 S fungal ribosomal genes from vesicular-arbuscular endomycorrhizal fungi colonizing roots. Appl. Environ. Microbiol. 58, 291-295 (1992).

\section{Acknowledgements}

We thank Mr. Xinquan Zhang for assistance during sample collection. We are grateful for Mao Lin's support in DNA extraction, amplification and sequencing.

\section{Author contributions}

X.R.Z. and L.D. designed the experiments. X.R.Z., L.D., G.F.X., and H.S.W. conducted the experiments and analyzed data. X.R.Z. and L.D. discussed data and results. X.R.Z. and G.F.X. wrote the manuscript.

\section{Competing interests}

The authors declare no competing interests.

\section{Additional information}

Correspondence and requests for materials should be addressed to X.R.Z.

Reprints and permissions information is available at www.nature.com/reprints.

Publisher's note Springer Nature remains neutral with regard to jurisdictional claims in published maps and institutional affiliations.

Open Access This article is licensed under a Creative Commons Attribution 4.0 International License, which permits use, sharing, adaptation, distribution and reproduction in any medium or format, as long as you give appropriate credit to the original author(s) and the source, provide a link to the Creative Commons licence, and indicate if changes were made. The images or other third party material in this article are included in the article's Creative Commons licence, unless indicated otherwise in a credit line to the material. If material is not included in the article's Creative Commons licence and your intended use is not permitted by statutory regulation or exceeds the permitted use, you will need to obtain permission directly from the copyright holder. To view a copy of this licence, visit http://creativecommons.org/licenses/by/4.0/.

(C) The Author(s) 2021 